\title{
Acute rhabdomyolysis during treatment with amisulpride and metformin
}

\author{
Francesco Ursini • Elena Succurro • \\ Alessandro Grembiale • Franco Arturi
}

Received: 11 September 2009 / Accepted: 27 November 2009/Published online: 18 December 2009

(C) Springer-Verlag 2009

Keywords Rhabdomyolysis · Creatine kinase .

Amisulpride $\cdot$ Metformin

\section{Background}

Rhabdomyolysis with increased serum creatine kinase (CK) is a potential side effect associated with the use of some medications, including statins and antipsychotics. Several cases of increased serum CK during or after treatment with atypical antipsychotics such as quetiapine, clozapine, or olanzapine have been reported [1-4]. Conversely, to our knowledge, no cases of rhabdomyolysis during metformin treatment are described in the literature. In this article we report a case of rhabdomyolysis during combined therapy with amisulpride and metformin, but not with either of these drugs alone.

\section{Case report}

Our patient is a 25 -year-old obese (BMI $\left.=32.2 \mathrm{~kg} / \mathrm{m}^{2}\right)$ male with hyperinsulinemia and a 4-year history of generalized anxiety disorder and dysthymia. He has been treated with benzodiazepines, SSRI, and tricyclic antidepressants without substantial improvement in psychiatric symptoms. For obesity he was previously treated with diet therapy achieving only a small reduction in body weight and in fasting insulin levels.

F. Ursini $(\bowtie) \cdot$ E. Succurro $•$ A. Grembiale $\cdot$ F. Arturi

Department of Experimental and Clinical Medicine,

University of Catanzaro "Magna Graecia",

Catanzaro, Italy

e-mail: francesco.ursini@yahoo.it
In May 2008, before starting any treatment, laboratory tests were performed. CK was normal $(110 \mathrm{IU} / \mathrm{L})$ and no other relevant alterations were found. In June 2008 metformin (500 mg three times a day) was started to control hyperinsulinemia and, together with a low-calorie diet, to achieve a greater reduction in body weight. After 2 weeks of therapy, routine laboratory tests were performed. CK remained in the normal range (126 IU/L) during the treatment with metformin.

After 3 weeks, on the advice of the psychiatrist, we decided to start amisulpride ( $200 \mathrm{mg}$ daily) for psychiatric complaints. After 2 weeks of therapy, biochemical analyses were performed for assessment of safety. All parameters were within normal range, except an elevated CK value of $1,962 \mathrm{IU} / \mathrm{L}$. No other abnormalities were evident and no symptoms and signs were present, so we decided to adopt a "watch and wait" strategy.

A few days later the patient started to complain of generalized muscular weakness, so routine blood tests were repeated. The screening showed a marked $\mathrm{CK}$ increase $(7,241 \mathrm{IU} / \mathrm{L}, \mathrm{CK}-\mathrm{MB}$ isoenzyme $<1 \%)$ with a concomitant increase in AST (95 IU/L), ALT (74 IU/L), aldolase (33 IU/L), and myoglobin $(139.3 \mathrm{ng} / \mathrm{mL})$. The patient did not report a history of intramuscular injections or trauma and performed no excessive physical activity in the week before admission.

Electromyography was performed with no evidence of pathologic findings. Muscular biopsy was performed, and there were no signs of muscular disease or enzymatic deficiency. Both drugs were suspended and another determination of serum CK was done. CK started to decline and returned back to normal after 3 weeks (105 IU/L). We decided to reintroduce amisulpride alone, the most likely drug to have caused CK elevation. After 1 week, 2 weeks, 
and 1 month of therapy, routine laboratory tests showed that the $\mathrm{CK}$ value remained within the normal range.

\section{Discussion}

Amisulpride, a substituted benzamide derivative, is an atypical antipsychotic used in the treatment of psychoses and, at lower doses (50-200 mg/daily), of dysthymia. It's a selective dopamine antagonist, with high affinity for D2 and D3 dopaminergic receptors. Like several medications of the same class, amisulpride can cause muscle toxicity in the form of neuroleptic malignant syndrome (NMS), by means of at least two different mechanisms. The first mechanism is the dopaminergic receptor antagonism in the hypothalamus, which could interfere with dopamine's normal role in central thermoregulation, leading to heat production and hyperthermia. The second mechanism is a direct toxicity on muscle cell membranes and an increased calcium release from the sarcoplasmic reticulum. In addition to NMS, isolated CK elevation without signs and symptoms of NMS has been also reported during treatment with atypical antipsychotics, such as quetiapine, clozapine, and olanzapine [3, 5-7]. We failed to find any report of isolated CK elevation with amisulpride, but several cases of NMS after or during treatment with this medication are described in the literature $[8,9]$.

Metformin is a biguanide oral hypoglycemic agent used to treat type 2 diabetes mellitus and hyperinsulinemia. It is the only biguanide available in Italy because phenformin, another biguanide, was withdrawn due to the high risk of lactic acidosis. Metformin can also cause lactic acidosis, even in patients taking therapeutic doses [10], via a mechanism not fully understood. It accumulates in the intestine, leading to an increased production of lactate, which lowers the $\mathrm{pH}$ within the liver and decreases lactate metabolism by suppressing pyruvate carboxylase. It also decreases glucose utilization and increases lactate production by the hepatocytes [11]. We did not find any reports of a direct link between metformin administration and rhabdomyolysis, but such a link has previously been reported with phenformin [12].

The close connection to the administration of both drugs together and the absence of other causes of rhabdomyolysis suggest that the elevated CK levels in this case resulted from the association of both drugs, and this explanation is supported by the fact that theoretically both drugs have the potential to cause muscle toxicity, especially in susceptible patients. While the exact mechanism of the CK elevation is unclear, it's possible that concomitant treatment with amisulpride and metformin caused damage to muscle membranes and a kind of muscle vulnerability to lowgrade injury, such as ordinary activity or mild exercise.

\section{Conclusions}

Our case report suggests that clinicians should be aware of this interaction and consider measuring $\mathrm{CK}$, particularly if any muscle-related symptoms appears.

\section{References}

1. Meltzer HY, Cola PA, Parsa M (1996) Marked elevations of serum creatine kinase activity associated with antipsychotic drug treatment. Neuropsychopharmacology 15:395-405

2. Apikoglu Rabus S, Izzettin F, Rabus M et al (2006) Severe creatine kinase increase during quetiapine and mirtazapine treatment. Psychopharmacology 185:263-264

3. Klein JP, Fiedler U, Appel H, Quante A, Jockers-Scherübl MC (2006) Massive creatine kinase elevations with quetiapine: report of two cases. Pharmacopsychiatry 39(1):39-40

4. Boot E, de Haan L (2000) Massive increase in serum creatine kinase during olanzapine and quetiapine treatment, not during treatment with clozapine. Psychopharmacology 150:347-348

5. Bachmann CJ, Nicksch B, de Lange D, Theisen FM, Remschmidt H (2007) Repeated creatine kinase elevation under treatment with quetiapine, clozapine, and aripiprazole in an adolescent. J Clin Psychopharmacol 27(6):710-1

6. Melkersson K (2006) Serum creatine kinase levels in chronic psychosis patients-a comparison between atypical and conventional antipsychotics. Prog Neuropsychopharmacol Biol Psychiatry 30(7):1277-82

7. Perlov E, Tebartz Van Elst L, Czygan M, Bubl E, Ebert D (2005) Serum creatine kinase elevation as a possible complication of therapy with olanzapine. Naunyn Schmiedebergs Arch Pharmacol 372(2):168-9

8. Abay E, Kose R (2007) Amisulpride-induced neuroleptic malignant syndrome. J Neuropsychiatry Clin Neurosci 19(4):488-9

9. Atbasoglu EC, Ozguven HD (2004) M, Goker C. Rhabdomyolysis and coma associated with amisulpride: a probable atypical presentation of neuroleptic malignant syndrome. J Clin Psychiatry 65(12):1724-5

10. Chang CT, Chen YC, Fang JT, Huang CC (2002) Metforminassociated lactic acidosis: case reports and literature review. J Nephrol 15:398-402

11. Lalau JD, Mourlhon C, Bergeret A, Lacroix C (1998) Consequences of metformin intoxication. Diabetes Care 21:2036-7

12. Yumul R, Steen SN, Osibamiro-Sedun A, Windokun A, Sapien R (2004) Rhabdomyolysis: a historical review with two illustrative cases. TraumaCare 14:143-7 